\title{
The Key Role of Multislice Computed Tomography for Direct Aor- tic Trans-catheter Aortic Valve Implantation
}

\section{Giuseppe Bruschi ${ }^{1 *}$, Fesc Paola Colombo ${ }^{1}$, Bruno Merlanti ${ }^{1}$, Diana Artioli ${ }^{2}$, Stefano Nava ${ }^{1}$, Francesco Soriano ${ }^{1}$, Oriana Belli ${ }^{1}$, Francesco Musca ${ }^{1}$, Luca Botta ${ }^{1}$, Giuseppe Tata $^{1}$, Ettore Colombo ${ }^{2}$, Fabrizio Oliva and Claudio F Russo ${ }^{1}$}

1 "De Gasperis" Cardiac Center, Niguarda Metropolitan Hospital, Milan, Italy

${ }^{2}$ Department of Radiology, Niguarda Metropolitan Hospital, Milan, Italy

*Corresponding author: Giuseppe Bruschi, "De Gasperis" Cardiac Center, Niguarda Metropolitan Hospital, Piazza dell'Ospedale Maggiore 3, 20162 Milan, Italy, Tel: +39-02-6444-2565, Fax: +39-02-6444-2566, E-mail: giuseppe.bruschi@fastwebnet.it

\begin{abstract}
Transcatheter Aortic Valve Implantation (TAVI) has been designed to treat elderly patients with severe aortic stenosis considered high-risk surgical candidates. The safety and effectiveness of TAVI have been demonstrated in numerous studies.

Retrograde femoral implantation is considered the less invasive approach for TAVI, however TAVI patients are also often affected by severe iliac-femoral arteriopathy, rendering the trans-femoral approach either unemployable or are deemed to carry a high risk of vascular injury.

Different arterial alternative access site has been used to implant transcatheter bioprosthesis, the subclavian, the ascending aorta and the carotid artery and more recently.

In the present report, we present indication and contraindication to direct aortic approach and we analyzed the key role of computed tomography.
\end{abstract}

\section{Keywords}

Catheter-based valve intervention, Aortic stenosis, Structural heart disease, Transcatheter technique, Multislice computed tomography

\section{Introduction}

Severe Aortic Stenosis (AS) is the most frequent form of valvular heart disease in western countries and a major cause of cardiovascular morbidity and mortality in the elderly. Symptomatic aortic stenosis has become an increasing health problem with a prevalence of $5 \%$ in patients over the age of 75 years [1,2].
Transcatheter aortic valves have been designed to treat patient affected by severe Aortic Stenosis (AS) deemed high risk for standard Aortic Valve Replacement (AVR). In fact, even if aortic valve replacement is the standard treatment for patients affected by AS [3], one third of patients, affected by severe aortic stenosis, may have been denied surgery [4]. This is related to the fact that the mortality rate associated with AVR increases substantially if multiple comorbidities are present such as: Significant left ventricular dysfunction, previous chest surgery or radiation, chronic obstructive pulmonary disease, liver or renal failure [5].

Since the first human percutaneous aortic valve implantation successfully performed by Cribier, et al. in 2002 more than 250,000 patients underwent TAVI worldwide with the two first CE mark trans-catheter aortic valve, the balloon-expandable Edwards SAPIEN prosthesis (Edwards Life sciences, CA, USA) and the self-expandable Medtronic Core Valve Revalving prosthesis (Medtronic Inc, MN, USA). A substantial body of data exists in relation to the clinical outcome of TAVI from post-marketing registries, observational clinical studies and National Registries [6-8] but and also form controlled randomized trial [9-11].

At the beginning of the experience the only access was the retrograde approach from the common femoral artery $[12,13]$. It must be considered that the trans-femoral approach requires favorable ileo-femoral arterial 
anatomy; this approach is contraindicated in patients with small size, severe calcifications and atherosclerosis of ileo-femoral arteries. To treat these patients a transapical approach with the Ascendra transapical catheter and the Edwards-Sapien valve was proposed [14]. However, this approach is more invasive since it requires direct Left Ventricle (LV) apex surgical exposure. Moreover, the transapical approach requires a dedicated antegrade delivery system. Since 2008 different alternative arterial access have been described, and received CE mark approval, for TAVI implantation in patient not suitable for peripheral femoral approach, the subclavian artery [15] and the direct aortic access [16,17]. More recently also a trans-carotid [18] and access has been describe [19].

\section{Direct Aortic Approach}

The procedure should be performed preferably in a hybrid operating room, with transesophageal echocardiographic and fluoroscopic guidance. To perform the operation, a multidisciplinary team involving interventional cardiologist, cardiac surgeon with expertise in hybrid procedure and wire skills, and is required.

The procedure is performed under general anesthesia, a double lumen endotracheal tube should be used for single left lung ventilation. A temporary pacing lead is advanced in the right ventricle through the femoral or jugular vein in patients without permanent pace-maker, to treat possible post-TAVI atrioventricular block. The radial artery or the best femoral artery is accessed by a single wall puncture under fluoroscopic guidance, to allow monitoring during the procedure and landmark aortic angiography through a 6-Fr pigtail. Heparin is administered to maintain an activated clotting time of $>250$ $\mathrm{s}$ throughout the procedure. Procedural details have been previously described [17,20-22]. The procedure should be performed through a right anterior mini-thoracotomy or through an upper mini sternotomy.

\section{The Role of Multislice Computed Tomography}

Trans-catheter candidates usually underwent as standard screening protocol, transthoracic echocardiogram, complete left heart catheterization, and coronary angiography, moreover all performed also chest and aortic-iliac-femoral computed tomography scan.

Multislice Computed Tomography (MSCT) is playing an increasingly important role in patient screening protocols before TAVI, provides detailed anatomic assessment of the aortic root and valve annulus assesses the suitability of iliofemoral access, and determines appropriate coaxial angles to optimize the valve implantation procedure. At minimum, an MSDCT scanner with 64-detectors technology is recommended for image acquisition. The specific scan protocols used for TAVI assessment vary, but typically include imaging of the entire aorta including the aortic root, thoracoabdominal aorta, and iliofemoral arteries. Because of the cyclic motion of the aortic valve, root, and ascending aorta, ECG-synchronized imaging of the root is critical to avoid image degradation secondary to motion artifacts. Two-options to achieve synchronization to the ECG are available, retrospective gating and prospective triggering. ECG-synchronization with retrospective gating acquires CT data throughout the entire cardiac cycle, and subsequently reconstructs images gated to a specific phase of the cardiac cycle. Alternatively, ECG-synchronization can be achieved with prospective triggering, which is associated with significantly lower radiation exposure [23].

On the basis of multislice computed tomography it is possible to evaluate:

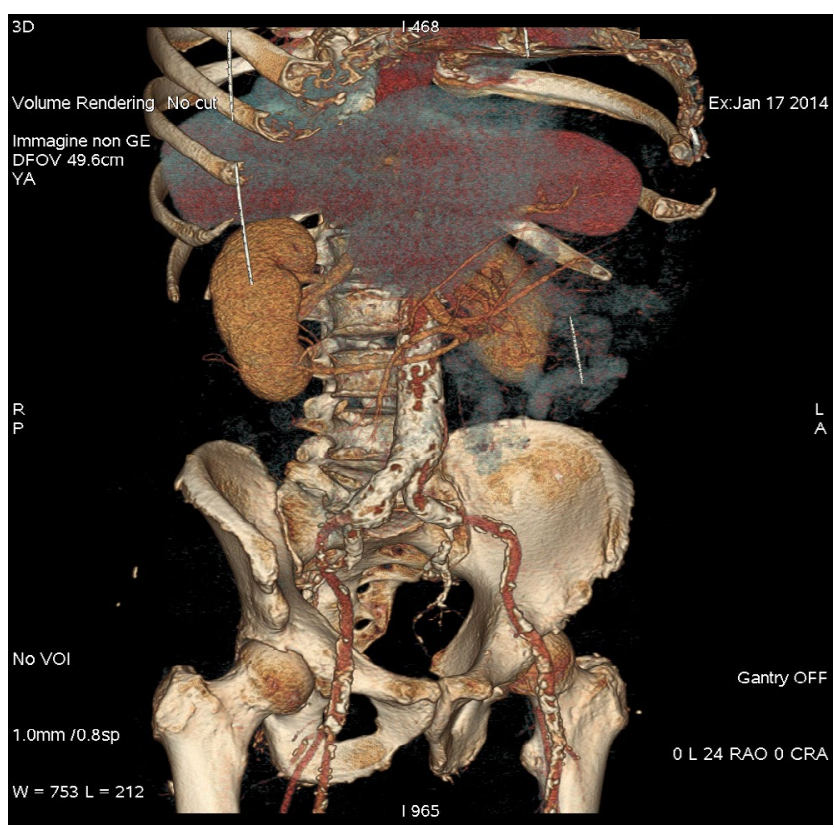

Figure 1: 3-d MSCT reconstruction allows to evaluate ileofemoral anatomy, tortuosity and calcifications.

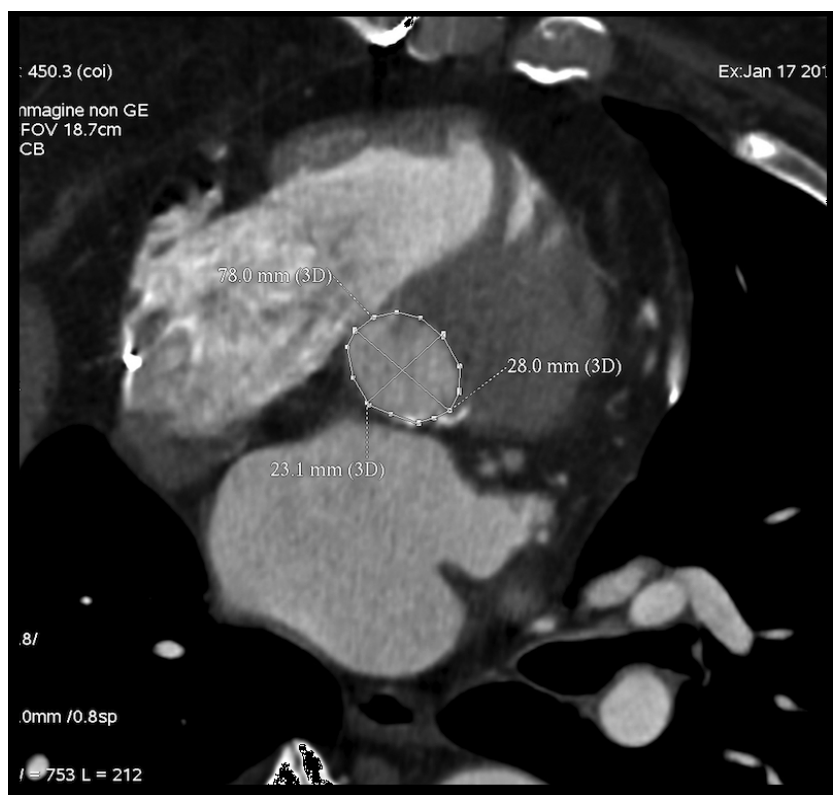

Figure 2: Double oblique transverse reconstruction of the aortic root below the commissural insertion of aortic valve allows to evaluate annulus size (perimeter, diameter). 
- lleo-femoral anatomy, vessel size, tortuosity and calcifications (Figure 1).

- Valve anatomy (bicuspid, tricuspid) location and degree of valve calcifications as well as annulus perim-

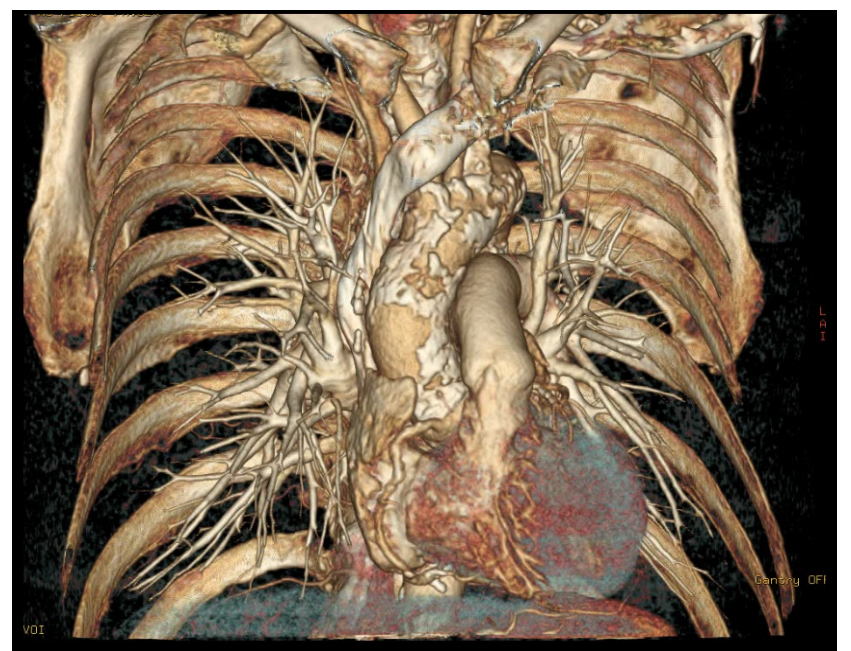

Figure 3: 3-d MSCT reconstruction of ascending aorta allows to evaluate ascending aorta anatomy, presence and location of calcification.

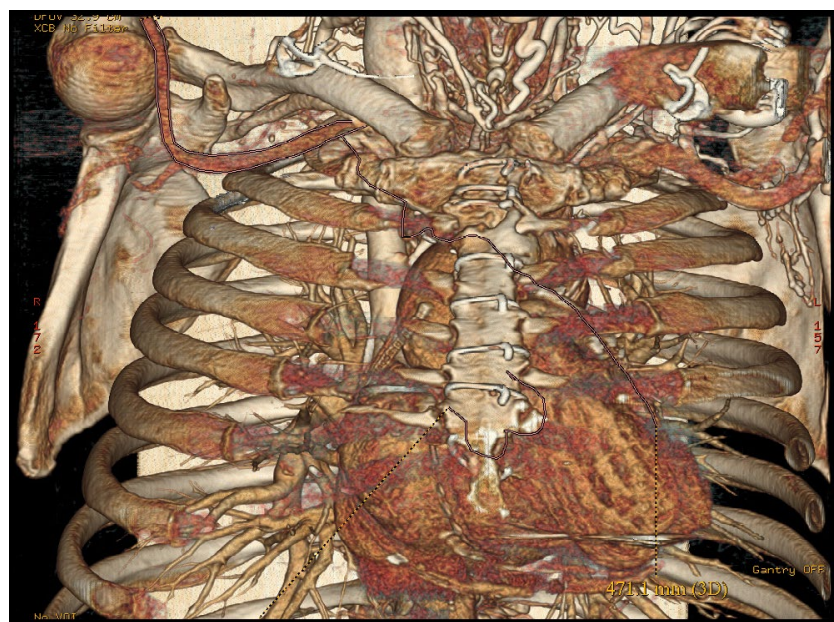

Figure 4: 3-d MSCT reconstruction of ascending aorta allows to evaluate the location of proximal graft anastomosis and location of internal mammary artery grafts. eter to choose the right TAVI size (Figure 2).

- Ascending aorta anatomy, presence and location of calcification, root angulation (Figure 3).

- Coronary height and the relationship between leaflet height and distance between annulus and coronary ostia, which identifies patients at risk for coronary occlusion during the TAVI procedure.

- In case of patient that underwent prior myocardial revascularization, the location of proximal graft anastomosis and location of internal mammary artery grafts (Figure 4).

- Evaluate coaxial trajectory from entry site on the ascending aorta to aortic annulus (Figure 5).

- Moreover, on the basis of pre-operative MCT it is possible to consider if right anterior mini-thoracotomy or upper-mini-sternotomy is more convenient for valve implantation (Figure 6).

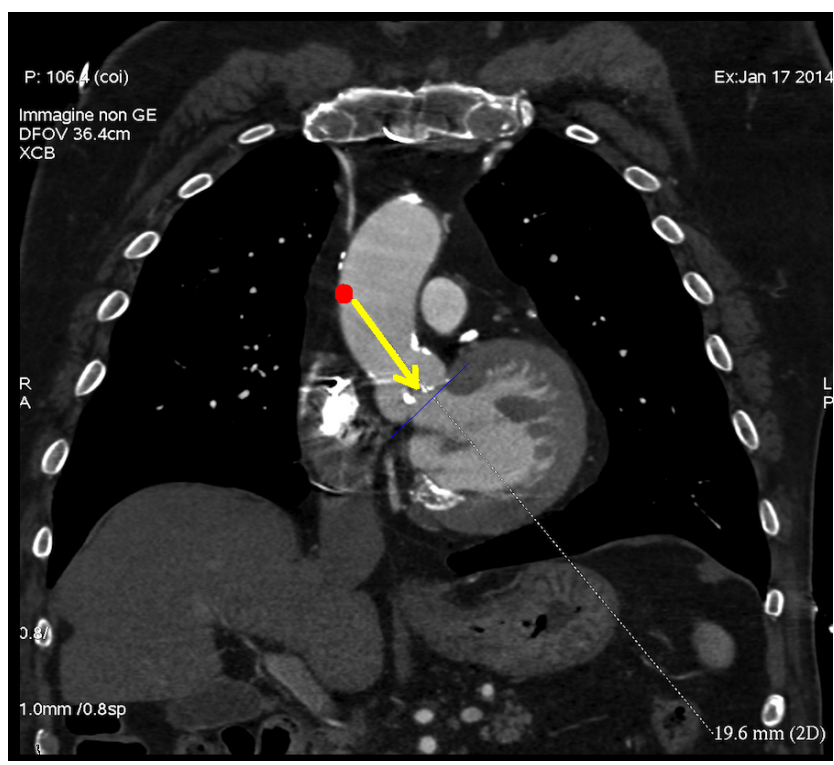

Figure 5: Multislice computed tomography allows to evaluate coaxial trajectory from entry site on the ascending aorta to aortic annulus in case of direct aortic approach.
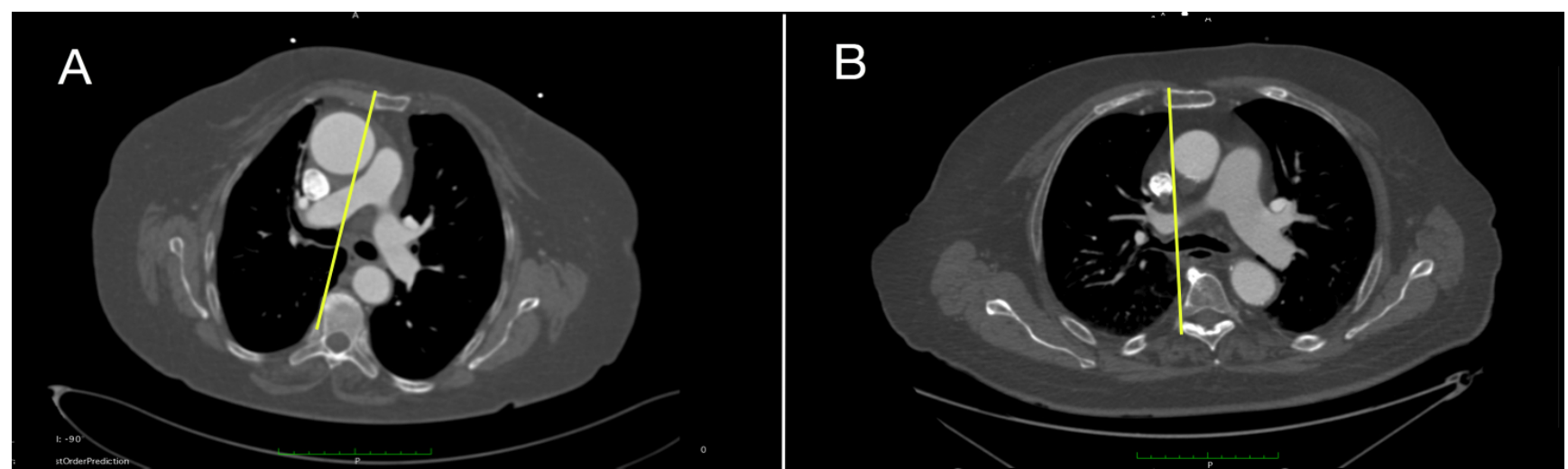

Figure 6: Cross-section image at the level of second costal cartilage allows to choose, in case of direct aortic approach, between right anterior thoracotomy or upper mini-sternotomy. Yellow line is drawn at the right edge of the sternum to determine percentage of aorta to its right; A) Example of a case suitable for right anterior thoracotomy; B) Example of a case suitable for mini-sternotomy. 
- Predict appropriate angle of deployment for trans-catheter valve implantation.

\section{Discussion}

Since the first case of a successful transcatheter implantation of an aortic bioprosthesis made by Cribier, et al. in April 2002 done via the femoral vein with an antegrade (trans-septal) access, trans-catheter aortic valve implantation has been successfully adopted in hundreds of centers across the world and more than 200,000 patients affected by severe aortic stenosis have been treated. The only access site during the initial phase was the femoral approach but during the years several different access routes have been developed (transapical, subclavian) and successfully adopted in many centers.

The subclavian/axillary approach was the first alternative approach that gained CE mark approval for CoreValve implantation on December 2010. Trans-subclavian/axillary approach has the advantage that axillary artery is easily accessible after surgical cut-down and that the procedure should be performed with local anesthetic and mild sedation. However the axillary is a fragile artery and patients are not eligible for the subclavian approach in case of vessel diameter $<6 \mathrm{~mm}$, heavy calcification, excessive tortuosity, or tight subclavian stenosis not amenable to percutaneous balloon angioplasty [24-26]. Moreover, the subclavian approach has to be considered cautiously in patients with patent left internal mammary artery graft.

Direct aortic approach gained CE mark on November 2011 , is theoretically more invasive as compared to axillary approach, requiring a mini-thoracotomy or upper mini-sternotomy. The advantage is that no limitation in vessel size is present and in severe horizontal aorta the procedure is feasible.

Direct aortic TAVI should be considered either a mirror image of the transapical procedure or a trans-femoral procedure through the aorta, with the special advantages of high control of valve deployment with no adverse effect on the left ventricle structure or function. Exposure of the aorta is a common skill for a cardiac surgeon; moreover, the majority of open heart operations are performed with cannulation of the aorta within two purse-string sutures, like in direct-aortic. For TAVI, the surgeon must be trained in the principles of catheterization techniques such as the Seldinger technique, wire exchanges and maintaining wire positions, shifting his focus from the surgical field to the imaging screen $[27,28]$.

The advantage of performing the implantation through a right anterior mini-thoracotomy is more evident in re-do patients, in whom a repeat sternotomy, even if partial, is a challenging procedure; indeed, right thoracotomy requires only limited dissection at the entry site on the ascending aorta and is feasible also in patients with patent venous grafts' proximal anastomosis on the ascending aorta [29].

This approach allows TAVI deployment to truly avoid the aortic arch contact, as there is neither a delivery system nor a wire placement in the arch, avoiding the risk of dislodging atherosclerotic plaque during valve passage, this may translate into lowering the incidence of stroke. The only contraindications for this approach are a complete porcelain aorta where there is presence of calcium at entry side zone and when the ascending aorta is not accessible.

All non-iliofemoral and proximal arterial accesses had the advantage of overcoming challenging aorto-ileo-femoral vascular disease, and avoids the risk of dislodging atherosclerotic plaque during valve passage through the aorta. The control of the delivery system is dramatically enhanced because any force is directly transmitted one-to-one without any loss of pushability and tractability compared with a TF approach. A co-axial trajectory to the aortic valve plane allows a perfect alignment of the TAVI device in the aortic annulus. This undoubtedly facilitates more accurate valve deployment, which might be particularly useful in patients with complex anatomical or pathophysiological conditions (horizontal ascending aorta, large annular sizes and primary aortic regurgitation) $[22,27,28]$.

Multislice computed tomography has assumed an increasingly important, complementary role before and after TAVI, and provides detailed anatomic assessment of the aortic root structures and iliofemoral access, adding to the information obtained with echocardiography and angiography. Therefore routine screening with MSCT is utilized by several groups to evaluate TAVI patients, and it is mandatory in patients evaluated for a direct aortic approach to improve the safety and potential application of TAVI in the management of symptomatic aortic stenosis patient considered high risk for surgery.

\section{Disclosure}

Dr. G. Bruschi is consultant for Medtronic and St. Jude-Abbott.

\section{References}

1. Lindroos M, Kupari M, Heikkila J, Tilvis R (1993) Prevalence of aortic valve abnormalities in the elderly: An echocardiographic study of a random population sample. J Am Coll Cardiol 2: 1220-1225

2. Nkomo VT, Gardin JM, Skelton TN, Gottdiener JS, Scott CG, et al. (2006) Burden of valvular heart diseases: A population-based study. Lancet 368: 1005-1011.

3. Vahanian A, Alfieri O, Andreotti F, Antunes MJ, Barón-Esquivias G, et al. (2012) Guidelines on the management of valvular heart disease (version 2012): the Joint Task Force on the Management of Valvular Heart Disease of the European Society of Cardiology (ESC) and the European Association for Cardio-Thoracic Surgery (EACTS). Eur J Cardiothorac Surg 42: S1-S44.

4. Lung B, Baron G, Butchart EG, Delahaye F, Gohlke-Bärwolf $C$, et al. (2003) A prospective survey of patients with 
valvular heart disease in Europe: the Euro Heart Survey on valvular heart disease. Eur Heart J 24: 1231-1243.

5. Charlson E, Legedza AT, Hamel MB (2006) Decision-making and outcomes in severe symptomatic aortic stenosis. J Heart Valve Dis 15: 312-321.

6. Piazza N, Grube E, Gerckens U, den Heijer P, Linke A, et al. (2008) Procedural and 30-day outcomes following transcatheter aortic valve implantation using the third generation (18 Fr) CoreValve revalving system: results from the multicentre, expanded evaluation registry 1-year following CE mark approval. Eurolntervention 4: 242-249.

7. Duncan A, Ludman P, Banya W, Cunningham D, Marlee D, et al. (2015) Long-term outcomes after transcatheter aortic valve replacement in high-risk patients with severe aortic stenosis: The U.K. Transcatheter Aortic Valve Implantation Registry. JACC Cardiovasc Interv 8: 645-653.

8. Gilard $M$, Eltchaninoff $H$, lung $B$, Donzeau-Gouge $P$, Chevreul K, et al. (2012) Registry of transcatheter aortic-valve implantation in high-risk patients. $\mathrm{N}$ Engl $\mathrm{J}$ Med 366: 1705-1715.

9. Leon MB, Smith CR, Mack M, Miller DC, Moses JW, et al. (2010) Transcatheter Aortic-Valve Implantation for Aortic Stenosis in Patients Who Cannot Undergo Surgery. N Engl J Med 363: 1597-1607.

10. Smith CR, Leon MB, Mack MJ, Miller DC, Moses JW, et al (2011) Transcatheter versus surgical aortic valve replacement in high-risk patients. N Engl J Med 364: 2187-2198.

11. Adams DH, Popma JJ, Reardon MJ, Yakubov SJ, Coselli JS, et al. (2014) Transcatheter aortic-valve replacement with a self-expanding prosthesis. N Engl J Med 370: 17901798.

12. Webb JG, Chandavimol M, Thompson C, Ricci DR, Carere RG, et al. (2006) Percutaneous aortic valve implantation retrograde from the femoral artery. Circulation 113: 842850.

13. Grube E, Buellesfeld L, Mueller R, Sauren B, Zickmann B, et al. (2008) Progress and current status of percutaneous aortic valve replacement: Results of three device generations of the CoreValve Revalving system. Circ Cardiovasc Interv 1: 167-175.

14. Ye J, Cheung A, Lichtenstein SV, Carere RG, Thompson CR, et al. (2006) Transapical aortic valve implantation in humans. J Thorac Cardiovasc Surg 131: 1194-1196.

15. Ruge $H$, Lange $R$, Bleiziffer S, Hutter A, Mazzitelli D, et al. (2008) First successful aortic valve implantation with the CoreValve ReValving System via right subclavian artery access: A case report. Heart Surg Forum 11: E323-E324.

16. Bauernschmitt $R$, Schreiber $C$, Bleiziffer $S$, Ruge $H$, Mazzitelli D, et al. (2009) Transcatheter aortic valve implantation through the ascending aorta: An alternative option for no-access patients. Heart Surg Forum 12: E63-E64.
17. Bruschi G, De Marco F, Fratto P, Jacopo Oreglia, Paola Colombo, et al. (2010) Direct aortic access through right minithoracotomy for implantation of self-expanding aortic bioprosthetic valves. J Thorac Cardiovasc Surg 140: 715717.

18. Stonier T, Harrison M, Choong AM (2016) A systematic review of transcatheter aortic valve implantation via carotid artery access. Int J Cardiol 219: 41-55.

19. Rodés-Cabau J, Puri R, Chamandi C (2017) The Caval-Aortic Access for Performing TAVR: Pushing the Limits of Alternative Access for Nontransfemoral Candidates. J Am Coll Cardiol 69: 522-525.

20. Bruschi G, De Marco F, Martinelli L, Klugmann S (2013) CoreValve transcatheter self-expandable aortic bioprosthesis. Expert Rev Med Devices 10: 15-26.

21. Rob Michiels (2010) CoreValve ReValving System for Percutaneous Aortic Valve Replacement. In: Serruys PW, Piazza N, Cribier A, Webb JG, Laborde JC, et al. Transcatheter aortic valve implantation- Tips and tricks to avoid failure. Informa Healthcare, New York, USA, 94-101.

22. Bapat V, G Bruschi (2013) Transaortic access is the key to success. Eurointervention 9: S25-S32.

23. Leipsic J, Gurvitch R, LaBounty TM, Min JK, Wood D, et al. (2011) Multidetector Computed Tomography in Transcatheter Aortic Valve Implantation. JACC Cardiovasc Imaging 4: $416-429$

24. Buschi G, Fratto P, De Marco F, Oreglia J, Colombo P, et al. (2010) The trans-subclavian retrograde approach for transcatheter aortic valve replacement: Single-center experience. J Thorac Cardiovasc Surg 140: 911-915, 915.e1-915.e2.

25. Petronio AS, De Carlo M, Bedogni F, Marzocchi A, Klugmann S, et al. (2010) Safety and efficacy of the subclavian approach for transcatheter aortic valve implantation with the CoreValve revalving system. Circ Cardiovasc Interv 3: 359-366.

26. Petronio AS, De Carlo M, Bedogni F, Maisano F, Ettori F, et al. (2012) 2-year results of CoreValve implantation through the subclavian access: A propensity-matched comparison with the femoral access. J Am Coll Cardiol 60: 502-507.

27. Hayashidaa K, Romano M, Lefèvre $T$, Chevalier B, Farge A, et al. (2013) The transaortic approach for transcatheter aortic valve implantation: A valid alternative to the transapical access in patients with no peripheral vascular option. A single center experience. Eur J Cardiothorac Surg 44: 692-700.

28. Bruschi G, De Marco F, Botta L, Barosi A, Colombo P, et al. (2015) Right anterior mini-thoracotomy direct aortic self-expanding trans-catheter aortic valve implantation: A single center experience. Int J Cardiol 181: 437-442.

29. Bruschi G, De Marco F, Botta L, Oreglia J, Colombo P, et al. (2012) Direct transaortic CoreValve implantation through right mini-thoracotomy in patients with patent coronary grafts. Ann Thorac Surg 93: 1297-1299. 